\title{
A Fuzzy Inference System for the Conjunctive Use of Surface and Subsurface Water
}

\author{
Liang-Cheng Chang, ${ }^{1}$ Hone-Jay Chu, ${ }^{2}$ and Yi-Wen Chen ${ }^{1}$ \\ ${ }^{1}$ Department of Civil Engineering, National Chiao Tung University, 1001 Ta Hsueh Road, Hsinchu 30050, Taiwan \\ ${ }^{2}$ Department of Geomatics, National Cheng Kung University, No. 1 University Road, Tainan 701, Taiwan
}

Correspondence should be addressed to Hone-Jay Chu; honejaychu@gmail.com

Received 8 May 2013; Accepted 29 June 2013

Academic Editor: Salvatore Sessa

Copyright (C) 2013 Liang-Cheng Chang et al. This is an open access article distributed under the Creative Commons Attribution License, which permits unrestricted use, distribution, and reproduction in any medium, provided the original work is properly cited.

\begin{abstract}
This study develops the water resources management model for conjunctive use of surface and subsurface water using a fuzzy inference system (FIS). The study applies the FIS to allocate the demands of surface and subsurface water. Subsequently, water allocations in the surface water system are simulated by using linear programming techniques, and the responses of subsurface water system with respect to pumping are forecasted by using artificial neural networks. The operating rule for the water systems is that the more abundant water system supplies more water. By using the fuzzy rule, the FIS conjunctive use model easily incorporates expert knowledge and operational polices into water resources management. The result indicates that the FIS model is more effective and efficient when compared with the decoupled conjunctive use and simulation-optimization models. Furthermore, the FIS model is an alternative way to obtain the conjunctive use policies between surface and subsurface water.
\end{abstract}

\section{Introduction}

The objective of the study is to develop a fuzzy rule-based method for the conjunctive use of surface and subsurface water systems. Zadeh [1] applied the fuzzy theory to mathematically deal with the imprecision and uncertainty. Fuzzy logic extends upon traditional Boolean logic and deals with the imprecision in human experience [2]. The fuzzy inference system (FIS) is an artificial intelligence technique that combines the fuzzy set, fuzzy logic, and fuzzy reasoning $[1,3-$ 6]. The FIS utilizes linguistic variables, fuzzy rules, and fuzzy reasoning and provides a tool for knowledge representation based on degrees of membership [7]. During the past decade, the FIS application ranged from runoff forecasting to surface water supply [3-6, 8, 9]. Shrestha et al. [3] developed a FIS to determine a real-world reservoir operation. They constructed a fuzzy rule-based model to derive operation rules for a multipurpose reservoir. Their research used reservoir storages, estimated inflows, and demands as the premises and took reservoir releases as the consequences. The finding showed that the fuzzy-based structure was ordinary and timesaving in computation. Russell and Campbell [4] developed the FIS for a simplified hydroelectric reservoir. The results also showed that fuzzy logic seemingly offered a way to improve the existing operating practices, which was relatively easy to explain and understand when compared with the complex optimization model. Panigrahi and Mujumdar [5] used a FIS for a reservoir operation model. The study incorporated expert knowledge for framing the fuzzy rule from an explicit stochastic model. Russell and Campbell [4] applied the Adaptive-Network-Based FIS (ANFIS) to water resources management and used the genetic algorithm to search the optimal reservoir operation based on a given inflow series. They used FIS for determining optimal water release according to reservoir depth and inflow. However, previous studies [3-5] mentioned that applying fuzzy logic to reservoir operation could remain limited to a single reservoir system.

Conjunctive use of surface and subsurface water is a challenging work for water resources management [10-14]. Conjunctive water management reduces the deficiencies by using subsurface water to supplement scarce surface water supply during the drought. The conjunction use enhances the reliability of water supplies by providing independent sources. Başaǧaoğlu and Mariño [10] developed 
a simulation-optimization model of a hypothetical river basin to determine optimal operating policies for jointly using surface and subsurface water supplies. The simulation model was the response function to incorporate the transient hydraulic interaction between stream and aquifer. The response function coefficients were derived from results of the numerical simulation model. Peralta et al. [11] employed simulation-optimization models to maximize total annual allocation of surface and subsurface water yield. They used the models in attempt to satisfy temporally increasing water needs for alternative future management scenarios. Philbrick and Kitanidis [12] proposed the gradient dynamic programming to solve the minimal operating cost problem by regarding surface and subsurface storages as state variables for realizing the impact of conjunctive use. Nishikawa [15] developed a simulation-optimization model for managing water resources for the city of Santa Barbara in a five-year planning horizon. Moreover, subsurface water simulation is linked with linear programming (LP). The model addressed the cost in water supply to meet demands and control seawater intrusion. Azaiez [16] developed the model for the conjunctive use of surface and subsurface water with an artificial recharge and integrated opportunity costs for the unsatisfied demand on the limitation of the final subsurface water level. The problem was simplified to be formulated as a convex program with linear constraints. Watkins and McKinney [14] applied decomposition algorithms to conjunctively managed surface and subsurface water systems, with reference to cost functions including both discrete and nonlinear terms. The complexity had mainly arisen from integrating surface and subsurface water, that is, two reservoirs and a confined aquifer. Their study incorporated the subsurface water system into the management model using the response matrix approach. However, many studies applied the artificial neural networks (ANNs) to model hydrology field complexity [17$22]$ including rainfall-runoff modeling $[22,23]$ and groundwater flow and transport [24]. The current work trained an ANN to predict the time-varying subsurface water level in response to management alternatives [18-21]. Coppola et al. [18] trained an ANN with MODFLOW simulation data to predict subsurface water levels at locations under various pumping conditions. The ANN forecasted subsurface water levels at the next time based on management alternatives including control and state variables at the current time.

Utilizing fuzzy rules, the FIS provides a tool to incorporate human expert experience for modeling a conjunctive use of surface and subsurface water. The FIS obtained allocated demand of ground and surface water in each stage simultaneously. Then, the simulator (i.e., LP and ANN) determined the future state of system, such as reservoir storages and hydraulic heads at the next time. Moreover, the LP simulated the operation of surface water system, and the ANN predicted hydraulic head variations under time-varying pumping.

\section{Methods}

Figure 1 illustrates the procedure of the FIS conjunctive use model. The FIS conjunctive use model comprises the control and simulation levels. Firstly, the FIS, which is in the control level, determines the assigned demand among surface and subsurface water each time step. After determining the assigned demands, the subsurface water submodel determines the hydraulic head using ANN [19], and the surface water allocation submodel obtains the reservoir supply and future reservoir storage using $\operatorname{LP}[25,26]$.

2.1. Fuzzy Inference System (FIS). The FIS is composed of five functional blocks [5]: (1) a rule base containing a number of fuzzy if-then rules; (2) a relational database which defines membership functions of the fuzzy set used in the fuzzy rules; (3) a fuzzification interface which transforms crisp inputs into degrees of match with linguistic values; (4) a fuzzy reasoning which performs inference operations on the rules; in FIS applications, the max-min and max product compositional operators are used most commonly because of their computational simplicity and efficiency; and (5) a defuzzification interface which transforms a combined output of fuzzy rules into a crisp value $[2,10,22]$. The current study uses the centroid method to defuzzify the aggregate fuzzy set, directly computing the real valued output as a normalized combination of membership values.

The study follows a typical process in developing the fuzzy system; for example, (1) define the linguistics variables; (2) construct the fuzzy rule structures; (3) determine the fuzzy set parameters; (4) encode the fuzzy sets, fuzzy rules, and the procedures; and (5) evaluate and tune the system [2] (Figure 2). In this study, the operation rule is the concept of water level index balance that the water system reaching the highest levels at the current time has a priority for supply at the next time [27, 28]. The FIS follows the following rule; that is, the abundant water system supplies more and the scarce water system supplies less water. In the study, the premises of the fuzzy rule are surface and subsurface water states, and the consequence is the assigned demand of each well. The $k$ th fuzzy rule in each time step is given as:

$$
\begin{aligned}
& \operatorname{if}\left(\sum_{i}\left(V_{i}^{t}+\mathrm{IF}_{i}^{t}\right) \text { is } A_{k}\right) \text { and }\left(\bar{h}^{t} \text { is } B_{k}\right) \\
& \text { then }\left(\bar{D}^{t} \text { is } C_{k}\right)
\end{aligned}
$$

where $V_{i}^{t}$ is the storage of the $i$ th reservoir; $\mathrm{IF}_{i}^{t}$ is the inflow of the $i$ th reservoir; $\bar{h}^{t}$ is the average subsurface water level in the observation wells; $\bar{D}^{t}$ is the assigned demand of each well in the subsurface water system; and $A_{k}, B_{k}$ and, $C_{k}$ are linguistic values in the $k$ th rule.

The premises and consequences are assigned as the triangular membership functions as in Figure 3. Two input variables are surface water state and subsurface water state, that is, average hydraulic head in the observations. The fuzzy membership functions of the inputs are divided into five categories, that is, "Low, Low Med., Medium, High Med. and, High." Triangular functions with equal base widths are the simplest possible, and these are often selected for practical applications [4]. In the study, the surface water state ranges 


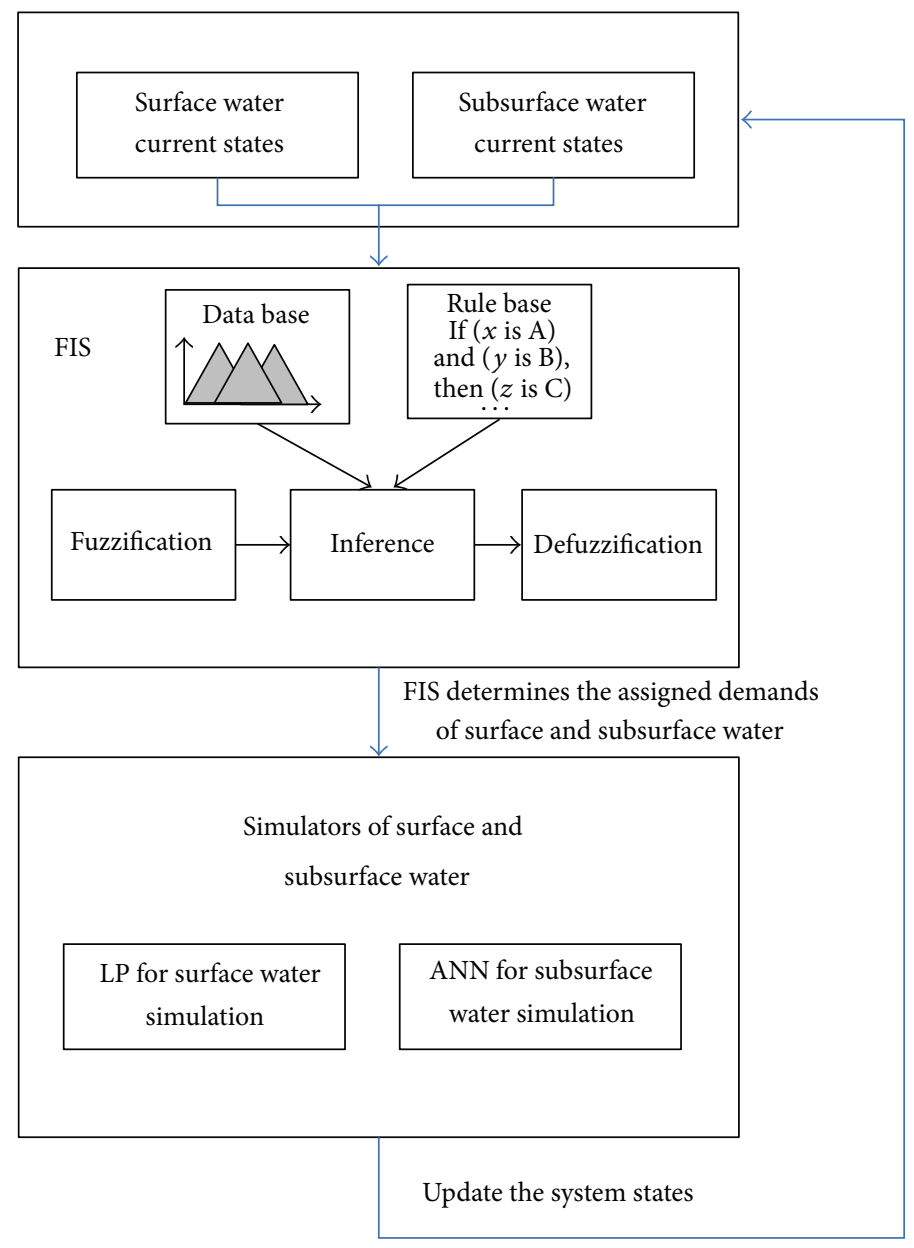

FIGURE 1: Flowchart of the FIS conjunctive use model.

from 0 to $120 \times 10^{6} \mathrm{~m}^{3}$ and the average hydraulic head ranges from 70 to $94 \mathrm{~m}$ in the observation wells. The output variable is the assigned demand of each well from subsurface water which ranges from 0 to $0.3 \mathrm{cms}$ and is divided into four categories in the membership function, that is, Low, Low Med., Medium, and High. The FIS computes the weights of each triggered rule, accumulating weights and outputs for each rule, and finally computing the weighted output for each rule [6]. Moreover, fuzzy sets provide a means of translating linguistic descriptors into a usable numerical form [26]. Table 1 shows the fuzzy rules; for example, If surface water state is Low, andsubsurface water level is High, then the assigned demand from subsurface water is High. After the FIS determines subsurface water demand, the surface water demand could be represented as

$$
\widehat{D}^{t}=D^{t}-\bar{D}^{t} \times N_{p}
$$

where $D^{t}$ is the whole water requirement in the $t$ th time step; $\widehat{D}^{t}$ is the surface water assigned demand in the $t$ th time step; and $N_{P}$ is the number of pumping wells.
2.2. Surface Water Submodel. The surface water allocation model is represented as

$$
\begin{array}{r}
J^{t}=\min _{X^{t}}\left[\sum_{j} c_{1, j} \mathrm{SH}_{j}^{t}+\sum_{i}\left(c_{2, i} G_{i, k}^{t}+c_{3, i} Z_{i}^{t}\right)\right], \\
t=1 \sim n ; i, k \in N_{S}, j \in N_{D}
\end{array}
$$

subject to

$$
\begin{gathered}
V_{i}^{t+1}=V_{i}^{t}+\mathrm{IF}_{i}^{t}-X_{i}^{t}-\mathrm{OF}_{i}^{t}, \quad i \in N_{S}, \\
V_{i}^{\min } \leq V_{i}^{t} \leq V_{i}^{\max }, \quad i \in N_{S}, \\
X_{i, j}^{\min } \leq X_{i, j}^{t} \leq X_{i, j}^{\max }, \quad i, j \in \Omega, \\
\sum_{i} X_{i, j}^{t} \leq \widehat{D}_{j}^{t}, \quad i \in \Omega, j \in N_{D}
\end{gathered}
$$

where $\mathrm{SH}_{j}^{t}$ is the shortage in demand $j$ at the $t$ th time step, $\mathrm{SH}_{j}^{t}=\widehat{D}_{j}^{t}-\sum_{i} X_{i, j}^{t} ; X_{i, j}^{t}$ means the supply from node $i$ in demand $j$ at time stept; and $N_{D}$ is the demand node set; in 


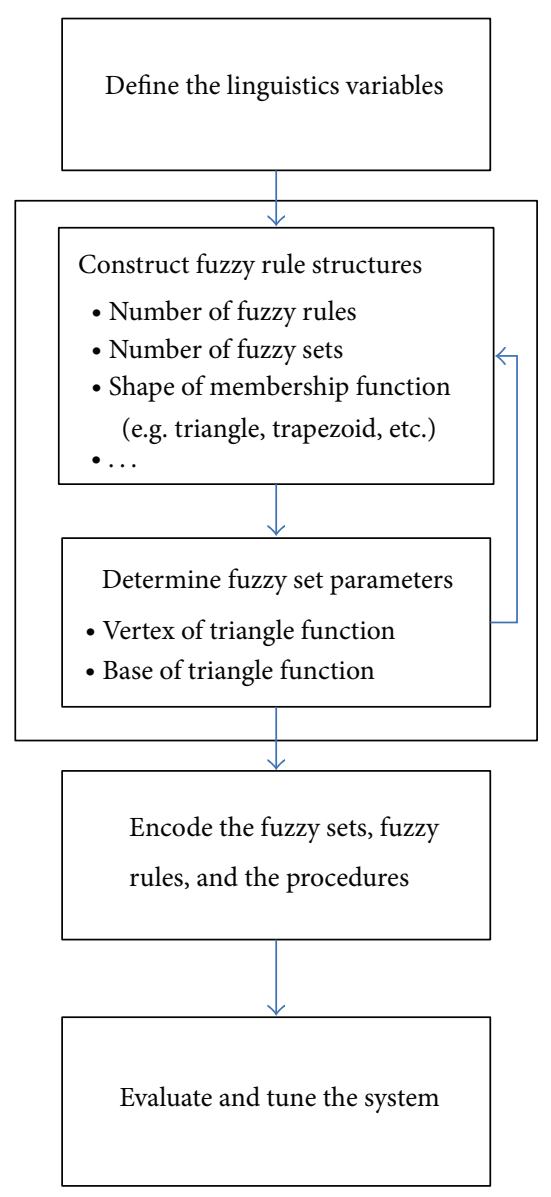

FIgURE 2: Procedure of developing the fuzzy system.

the study, $j=1 . G_{i k}^{t}$ is the difference between water level index (WLI) of the reservoirs $i$ and $k$ at the $t$ th time step [27, 28] and $N_{S}$ is the reservoir storage node set; if $i=k$, then $G_{i k}^{t}=0$ otherwise. $Z_{i}^{t}$ is the ratio of residual volume to the capacity in reservoir $i$ at the $t$ th time step: $\left(V_{i}^{\max }-V_{i}^{t}\right) / V_{i}^{\max } ; c_{1}, c_{2}$, and $c_{3}$ are the weight coefficients applied to the shortage, surfacewater level index difference, and residual reservoir volume ratio, respectively $\left(c_{1}=1, c_{2}=10\right.$, and $\left.c_{3}=1\right)$. $X_{i}^{t}$ denotes the release from reservoir $i$ at time stept; OF $_{i}^{t}$ denotes spills of $i$ reservoir at time step $t$; and $V_{i}^{t}$ is the storage of the reservoir i. $V_{i}^{\min }$ and $V_{i}^{\max }$ are minimum and maximum capacity of the reservoir $i$. $X_{i, j}^{\min }$ and $X_{i, j}^{\max }$ are minimum and maximum discharge of the pipe from node $i$ to $j$ and $\Omega$ is the node set of the system network. The surface water demand considers hedging rule at time step $t$. The hedging rule is illustrated as follows:

$$
\begin{gathered}
\text { if } V_{\text {joint }}^{t} \geq V_{2}, \quad \text { then } \widehat{D}^{t}=\widehat{D}^{t}, \\
\text { if } V_{2}>V_{\text {joint }}^{t} \geq V_{3}, \quad \text { then } \widehat{D}^{t}=\omega_{1} \widehat{D}^{t} \\
\text { if } V_{\text {joint }}^{t}<V_{3}, \quad \text { then } \widehat{D}^{t}=\omega_{2} \widehat{D}^{t},
\end{gathered}
$$

where $V_{\text {joint }}^{t}$ is the joint storage in all reservoirs; $V_{1}$ is the sum of maximum storage (upper limit) among the reservoirs; $V_{2}$ is
TABLE 1: Rule table for the operation in conjunctive use operation using FIS within high usage of subsurface water.

\begin{tabular}{lccccc}
\hline \multicolumn{6}{c}{ Surface water state } \\
& Low & Low Med. Medium & High Med. High \\
\hline Subsurface water state \\
Low & Low & Low & Low & Low & Low \\
Low Med. & High & High & Low & Low & Low \\
Medium & High & High & Low & Low & Low \\
High Med. & High & High & Low & Low & Low \\
High & High & High & Low & Low & Low \\
\hline
\end{tabular}

the sum of target storage (lower limit) among the reservoirs; and $V_{3}$ is the sum of firm storage (critical limit) among the reservoirs; $\omega_{1}$ and $\omega_{2}$ are the rationing factors. In the study, $\omega_{1}$ and $\omega_{2}$ are 0.85 and 0.75 .

This study uses the linear programming (LP) to simulate the surface water system in (3) (8). The formulation is as follows. Equation (3) specifies the objective function consisting of three subobjectives which include the total shortage in each time step, the difference between the reservoir water level, and the ratio of the residual water volume to the capacity of each reservoir. Equations (4) and (5) list the mass balance equation and the demand constraint of each reservoir in each time step. Equation (6) specifies the capacity constraints for each reservoir in each time step. Equations (7) and (8) specify the constraints on the flow through the pipes and hedging rule in each time step. In the study, the model first determines the demand with the hedging rule (8), and then the LP determines the flows in the system at $t$ while satisfying the demand with the hedging rule.

2.3. Subsurface Water Submodel. The current study uses ANN to serve as the simulator for modeling nonlinear and time-varying groundwater flow. An ANN consists of a number of neurons arranged in an input layer, an output layer, and one hidden layer. The inputs are state vectors, which are the set of hydraulic head $\left(h^{t}\right)$ and the pumping rate vector $\left(P^{t}\right)$. The output is the hydraulic head vector at the next time step $\left(h^{t+1}\right)$. The subsurface water model is illustrated as follows:

$$
\begin{gathered}
h^{t+1}=f\left(h^{t}, P^{t}\right), \\
P_{i}^{t} \leq \bar{D}^{t},
\end{gathered}
$$

where $h^{t}$ and $h^{t+1}$ are subsurface water level vectors at time $t$ and $t+1 ; P^{t}$ is the supply vector offered from the pumping well; and $P_{i}^{t}$ is the supply at the well $i$ in the th time step. Equation (9) represents the subsurface water transient equation with the artificial neural networks. Equation (10) represents the demand-supply of constraint each well at time $t$. Pumping rate of each well is assumed to be less than or equal to assigned demand with the FIS in the study.

The ANN was trained by the back-propagation learning algorithm [29] for subsurface water table prediction. The typical processes of the ANN parameters identification such as the number of hidden layers and the neurons are listed 
in Negnevitsky [2]. This ANN consists of a three-layer feedforward network and one hidden layer in which the layer contains twenty neurons.

2.4. The Simulation-Optimization Model. To compare the FIS with the simulation-optimization model, this study further formulates the problem (1) (10) into the sequential optimization problem. As previously stated, this investigation integrates sequential optimization and simulation models to solve the problem defined as follows:

$$
\begin{array}{r}
J^{t}=\min _{X^{t}, P^{t}}\left[\sum_{j} c_{1, j} \mathrm{SH}_{j}^{t}+\sum_{i}\left(c_{2, i} G_{i, k}^{t}+c_{3, i} Z_{i}^{t}\right)+c_{4} S G^{t}\right], \\
t=1 \sim n ; i, k \in N_{S}, j \in N_{D}
\end{array}
$$

subject to $(4) \sim(10)$,

where $S G^{t}$ represents the difference between WLI of surface and subsurface water systems at time step $t$ and $c_{4}$ is the weight coefficient applied to WLI difference between surface and subsurface water systems $\left(c_{4}=10\right)$. In the model, the pumping rates are first obtained by the heuristic optimization (i.e., genetic algorithm (GA)). Then, the release of each reservoir and next time-step states are determined by (4) (10) using the LP and ANN.

2.5. Case Study. This study performs numerical analysis on a water supply problem to verify effectiveness of the proposed methodology. The planning horizon in the study is twenty years and each time step is ten days. Figure 4 shows the conjunctive use system including two reservoirs and an aquifer. Reservoirs 1 and 2 contain a capacity of $7.0 \times 10^{7}\left(\mathrm{~m}^{3}\right)$ and $5.0 \times 10^{7}\left(\mathrm{~m}^{3}\right)$. Reservoir operation rules will be designed to vary with periods [30]. Figures 5(a) and 5(b) are operation rule curves for Reservoirs 1 and 2, respectively. Full of water in reservoirs is assumed as the initial condition. Figure 6 shows both reservoirs inflows that reflect the hydrological dynamics of Taiwan. The inflow ratio of drought season to wet season is 0.32 (Reservoir 1) and 0.20 (Reservoir 2). Moreover, the capacity factor (i.e., effective capacity/average annual flow) of Reservoir 1 is 0.24 while that of Reservoir 2 is 0.35 . Figure 7 demonstrates a hypothetical homogeneous unconfined aquifer with dimensions of $17 \mathrm{~km}$ by $17 \mathrm{~km}$. The case contains $170 \times 170$ finite-difference meshes along with five pumping wells (red blocks) and five observation wells (black blocks). The boundary conditions on the north and south sides are no-flow boundaries. The west and east constant-head boundaries are $100 \mathrm{~m}$ and $80 \mathrm{~m}$, respectively. Aquifer properties and simulation parameters are shown in Table 2.

\section{Results and Discussion}

3.1. Comparison of Decoupled and Coupled Conjunctive Use Operations. Table 3 demonstrates the series of models under the same water requirement amount, $1.5 \times 10^{7}\left(\mathrm{~m}^{3} /\right.$ tenday). Case 1 considers decoupled operation that surface
TABLE 2: Aquifer properties and simulation parameters.

\begin{tabular}{lc}
\hline Parameter & Value \\
\hline Aquifer thickness $(\mathrm{m})$ & 110 \\
Specific yield & 0.2 \\
Porosity & 0.2 \\
Horizontal hydraulic conductivity $(\mathrm{m} / \mathrm{s})$ & 0.0001 \\
Vertical hydraulic conductivity $(\mathrm{m} / \mathrm{s})$ & 0.0001 \\
Simulation time step length $($ days $)$ & 10 \\
Maximum pumping rate $(\mathrm{cms})$ & 0.3 \\
\hline
\end{tabular}

TABLE 3: Case abstract in the study.

\begin{tabular}{ll}
\hline $\begin{array}{l}\text { Case } \\
\text { number }\end{array}$ & Description \\
\hline 1 & $\begin{array}{l}\text { Decoupled conjunctive use operation } \\
\text { Conjunctive use operation using FIS within high usage } \\
\text { of subsurface water }\end{array}$ \\
3 & $\begin{array}{l}\text { Conjunctive use operation using a } \\
\text { simulation-optimization model } \\
\text { Conjunctive use operation using FIS within low usage } \\
\text { of subsurface water }\end{array}$ \\
\hline
\end{tabular}

water is supplied in advance, and subsurface water is then supplied. Case 2 considers the conjunctive use of surface and subsurface water simultaneously by using FIS based on fuzzy rules (Table 1). Table 4 presents the water supply and shortage index (SI) $[31,32]$ which, proposed by the US Army Corps of Engineers, could represent the severity of the long-term water shortage from surface and subsurface water. The result shows that the 10-day and annual SI of Case 2 are lower than those of Case 1. It implies that the FIS could improve water shortage in the case study. Compared with Case 1, Case 2 decreases the shortage by $26.23 \%$, and the FIS makes a significant drop in deficit risk. This indicates that the FIS conjunctive use of surface and subsurface water is more efficient. The FIS specifies how much water is supplied from surface and subsurface water to achieve system demand requirement. According to the fuzzy rules, water is supplied from surface water in normal time and from subsurface water during the drought. Figure 8 implies the relationship between subsurface water supply and shortage in decoupled and coupled conjunctive use models (Cases 1 and 2). Furthermore, subsurface water is supplied earlier in coupled conjunctive use model (Case 2) than in the decoupled conjunctive use model (Case 1) during the drought. The result indicates that timing of water allocation is significantly important. Considering the FIS conjunctive operation (Case 2), water supply from subsurface water in advance may reduce the impact of shortage under a long-term operation. Ponnambalam et al. [33] compared general operating rules developed by both fuzzy rules and regression-based rules. Their results demonstrate that the FIS rules perform much better than the regression rules for dealing with uncertainty of inflows. Fuzzy sets provide a nonfrequentist approach to considering uncertainty [34]. The FIS conjunctive use of surface and subsurface water can enhance the reliability of water supplies by providing 
TABLE 4: The shortage indices and the supply of water system in the cases.

\begin{tabular}{|c|c|c|c|c|c|}
\hline \multicolumn{2}{|c|}{ Case } & 1 & 2 & 3 & 4 \\
\hline \multicolumn{2}{|c|}{ 10-day SI } & 2.09 & 1.44 & 1.50 & 1.84 \\
\hline \multicolumn{2}{|c|}{ Annual SI } & 0.61 & 0.45 & 0.48 & 0.59 \\
\hline \multicolumn{2}{|c|}{ Number of 10 days in shortage } & 262 & 234 & 241 & 250 \\
\hline \multirow{2}{*}{ Surface water $\left(10^{4} \mathrm{~m}^{3}\right)$} & 10-day supply & 1345.25 & 1345.07 & 1343.68 & 1347.77 \\
\hline & Annual supply & 48429.10 & 48422.62 & 48372.49 & 48519.6 \\
\hline \multirow{2}{*}{ Subsurface water $\left(10^{4} \mathrm{~m}^{3}\right)$} & 10-day supply & 49.77 & 73.44 & 65.60 & 56.80 \\
\hline & Annual supply & 1791.56 & 2643.77 & 2361.48 & 2044.84 \\
\hline
\end{tabular}

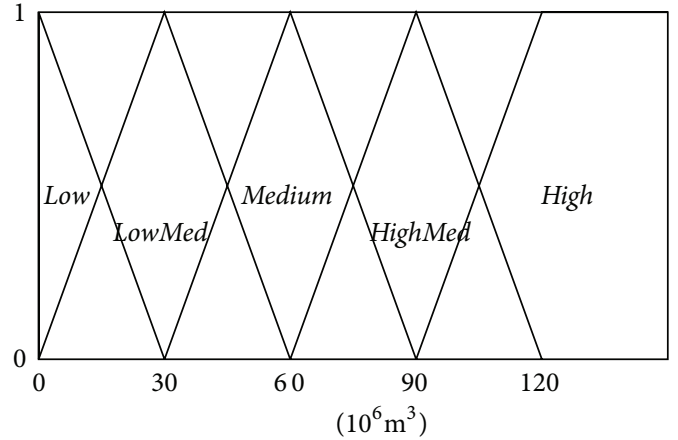

(a)

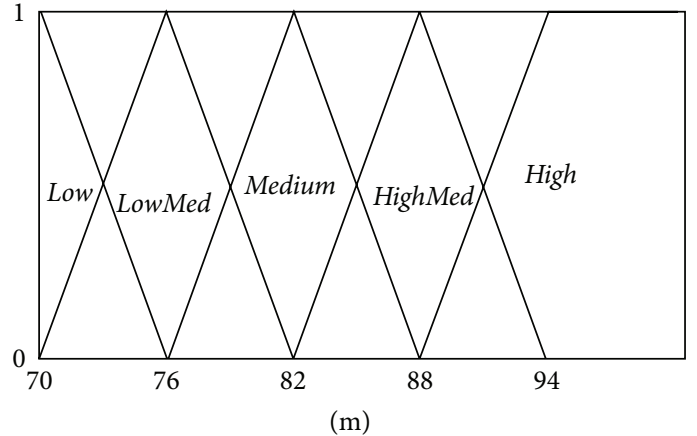

(b)

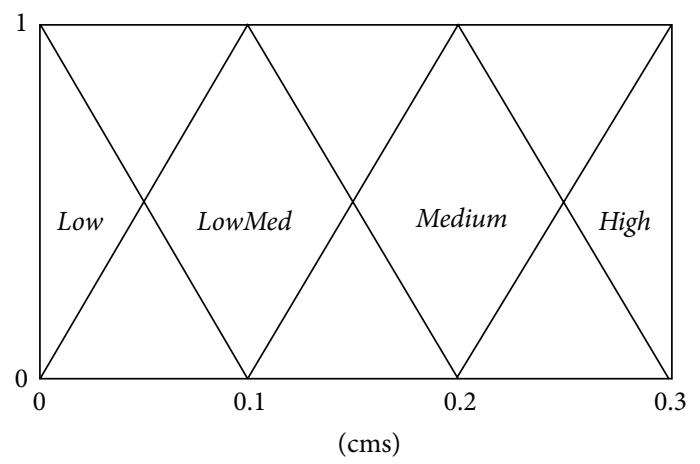

(c)

FIGURE 3: Fuzzy membership function for (a) input 1: surface water state, (b) input 2: subsurface water state, and (c) output: pumping rate.

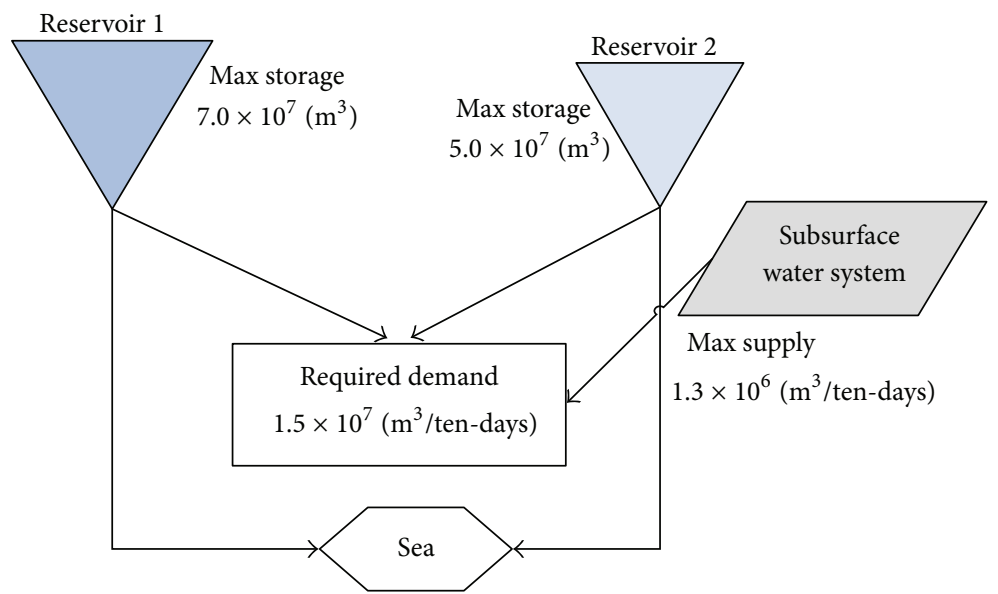

FIGURE 4: Schematic diagram of water resources system. 


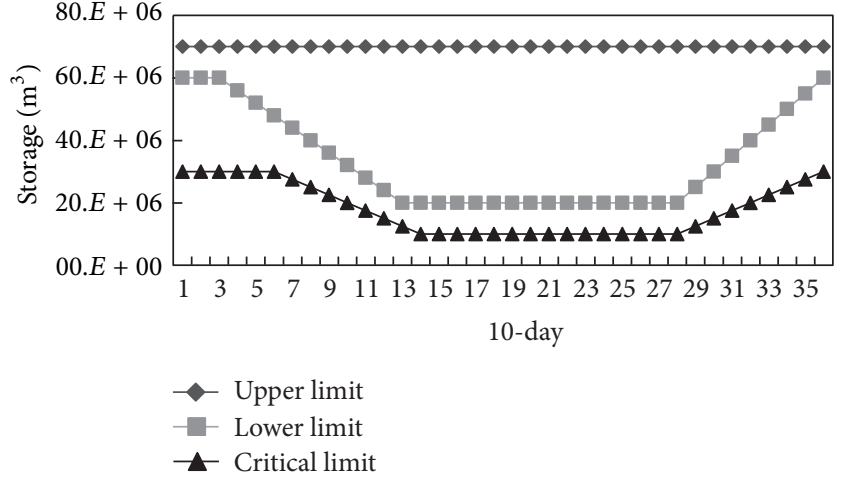

(a)

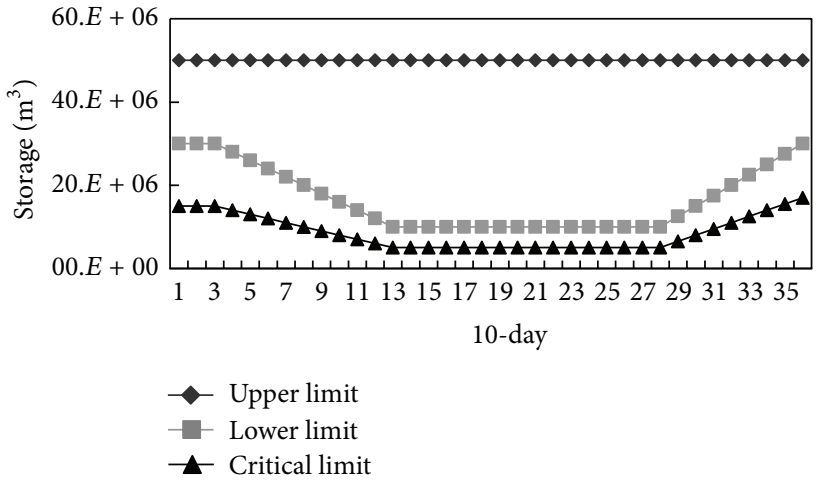

(b)

FIgURE 5: The operation rule curve of (a) Reservoir 1 and (b) Reservoir 2.

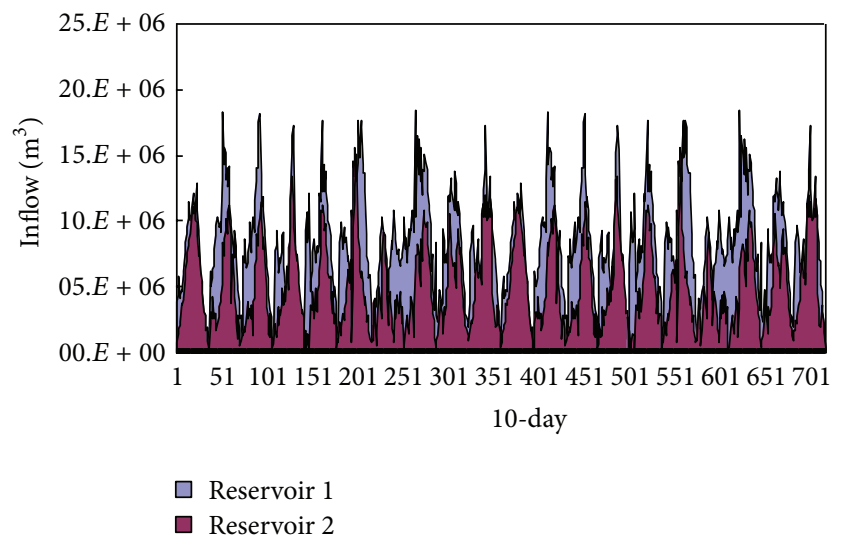

FIGURE 6: Reservoir inflows in surface water system.

independent sources. Surface and subsurface water systems contain distinctly different characteristics; for example, surface water is rapid fluctuations and subsurface water varies gradually. Considering the fuzzy rules that abundant water system supplies more water, the FIS is efficiently applicable to the management of surface and subsurface water.

\subsection{Comparison of FIS and Simulation-Optimization Model.} This study compares the FIS and simulation-optimization models to verify effectiveness of the proposed FIS methodology for conjunctive use of surface and subsurface water. Simulation-optimization approach is used in Case 3 for minimizing the water shortage and balancing the usages between surface and subsurface water (11). In the simulationoptimization model, the surface and subsurface water simulation models including the LP and ANN are embedded in the genetic algorithm (GA) to determine the pumping rate and water allocation in each time step sequentially. For the details of GA refer to McKinney and Lin [35], Chen et al. [36], and Chang et al. [37].

The result reveals that 10-day and annual SI of the FIS and simulation-optimization model (Cases 2 and 3) are similar (Table 4). Both models can decrease the water shortage more than $20 \%$ in comparison Case 1 . This information allows the

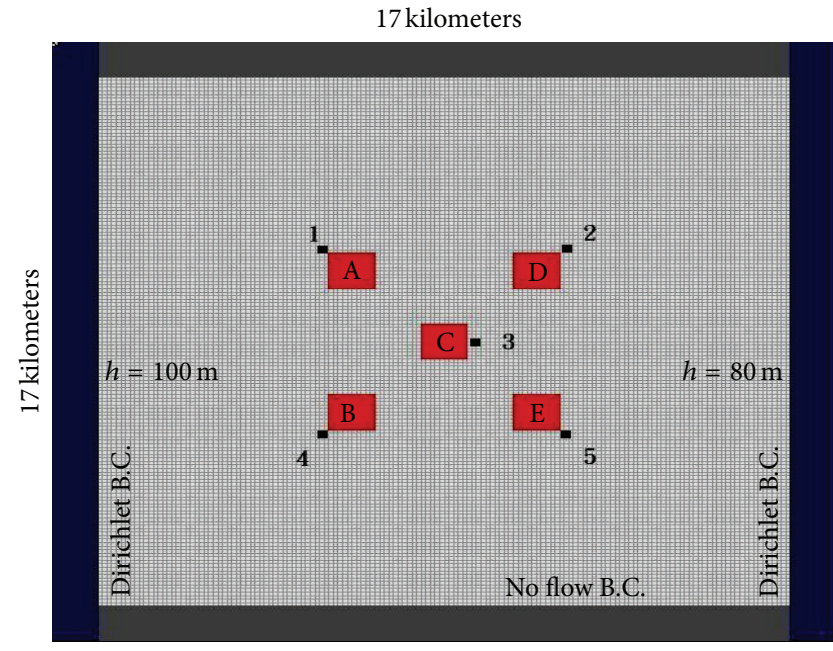

(Red blocks mean pumping well clusters and black blocks mean observation wells)

FIGURE 7: Model of subsurface water system, observation, and pumping wells.

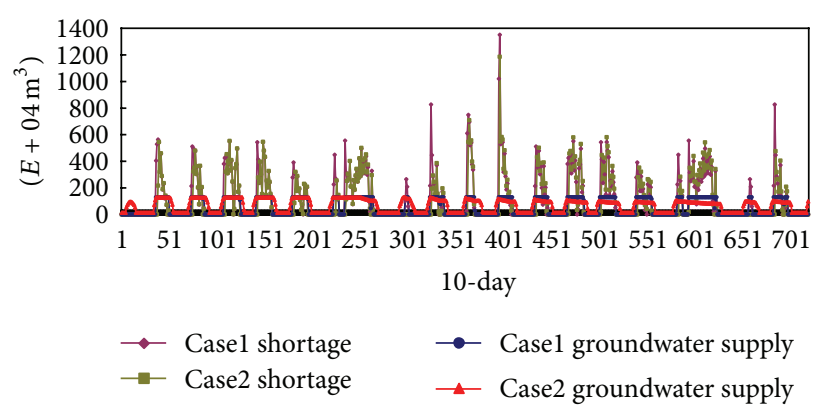

FIGURE 8: Comparison of supply of subsurface water and shortage between Cases 1 and 2 .

decision makers to control water supply for a long term by the FIS. Similar to the simulation-optimization model, the fuzzy operating rules specify how water is managed throughout the system to achieve system demand requirement. During 
TABLE 5: Validation results in subsurface water ANN model.

\begin{tabular}{lccc}
\hline & MSE $\left(\mathrm{m}^{2}\right)$ & RMSE $(\mathrm{m})$ & AME $(\mathrm{m})$ \\
\hline Obs. 1 & 0.42 & 0.65 & 0.51 \\
Obs. 2 & 0.45 & 0.67 & 0.53 \\
Obs. 3 & 1.04 & 1.02 & 0.82 \\
Obs. 4 & 0.43 & 0.65 & 0.52 \\
Obs. 5 & 0.47 & 0.69 & 0.54 \\
\hline
\end{tabular}

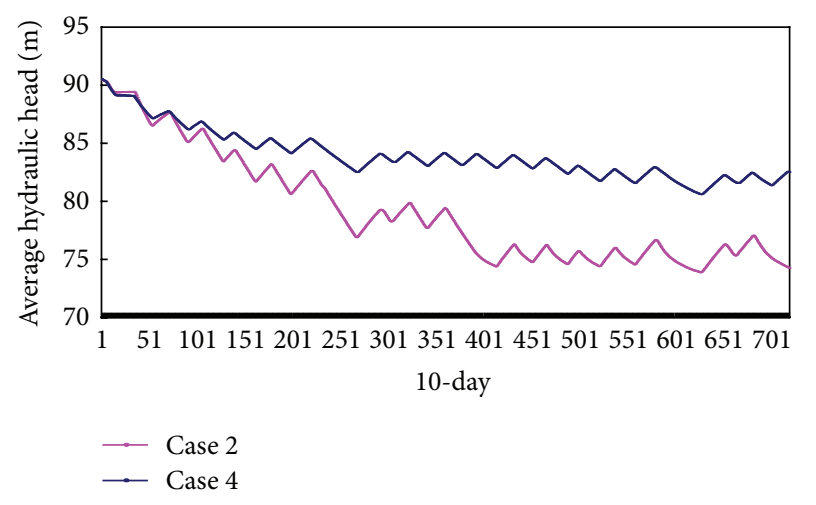

FIgURE 9: Comparison of average hydraulic heads in Case 2 and 4.

the drought, subsurface water is used in advance, and surface water is saved. Water supply using the FIS will reduce the impact of shortage. However, problem solving requires hundreds to thousands of numerical simulation runs for searching water supply strategies in the GA approach. For example, the maximum number of generations is twenty, and the population size for each generation is fifty chromosomes. Therefore, 1000 searching possibilities at most are needed in each time step. The computation is more effective using the FIS than the GA. However, the FIS approach obtains near-optimal solutions and saves considerable computational time. The FIS provides an alternative way for conjunctive operations that offer the good chances for water supply management [3-5]. The FIS is easy to apply and extend to a complex water system [3], utilized in controlling humanistic systems in water resources management, and offers an alternative way for conjunctive operation.

\subsection{Subsurface Water Table Simulation by ANN and Control} by FIS. The ANN inputs include the hydraulic heads in five observation wells and pumping rates in five pumping wells at current time, and the outputs are hydraulic heads in the observations at next time. The data are generated by the MODFLOW and sets of input-output patterns are generated by a random pumping rate between the minimum and maximum (from 0 to $0.3 \mathrm{cms}$ ). The MODFLOW, is a physical finite-difference numerical flow model and a computer program developed by the US Geological Survey [38]. Moreover, a network training function updates weight and bias values according to Levenberg-Marquardt optimization [39]. The transfer functions with a hidden layer and the output layer are hyperbolic tangent. If the training stop criteria (i.e., MSE = $10^{-7}$ ) are not met, the learning algorithm continues.
TABLE 6: Rule table for the operation in conjunctive use operation using FIS within low usage of subsurface water.

\begin{tabular}{lccccc}
\hline \multicolumn{5}{c}{ Surface water state } \\
& Low & $\begin{array}{c}\text { Low } \\
\text { Med. }\end{array}$ & Medium & $\begin{array}{c}\text { High } \\
\text { Med. }\end{array}$ & High \\
\hline Subsurface water state & & & & & \\
Low & Low & Low & Low & Low & Low \\
Low Med. & Medium & Medium & Low & Low & Low \\
Medium & Medium & Medium & Low & Low & Low \\
High Med. & Medium & Medium & Low & Low & Low \\
High & Medium & Medium & Low & Low & Low \\
\hline
\end{tabular}

The total available data has been divided into two sets, training and validation sets: 2,500 samples were used to train the ANN, and 1,000 samples were used for validation. Table 5 presents the ANN validation results. Accuracy of the ANN model can be quantified when compared with MODFLOW. Comparing relative errors reveals that, among the table, Observation 1 is the lowest error in the estimation. Results demonstrate that relative error with respect to average subsurface water level is $1.3 \%$ or less. After the validation, the ANN simulates the subsurface water level with time behind the FIS operating. Accordingly, the ANN obtains better results, and the computing time of the ANN model is about $1 / 53$ of a traditional MODFLOW. This result reveals that the ANN predicts hydraulic heads efficiently at the selected control locations under variable pumping but condensed surrogate for subsurface water flow model in interesting cells $[18,21]$. Results show the ANN approach has a great potential to predict subsurface water level because it permits developing complex and nonlinear models.

Figure 9 shows time-varying hydraulic heads in the two FIS cases (Cases 2 and 4) under various pumping strategies. The fuzzy rules for high and low usages in Cases 2 and 4 are represented in Tables 1 and 6 . Overall, the average hydraulic heads vary with dry-wet cycles. In Cases 2 and 4, a fuzzy rule-based system determines the pumping rate considering hydraulic head constraint implicitly. Moreover, the FIS will decide to pump a large volume of subsurface water in Case 2 and pump a small volume of subsurface water in Case 4; therefore the hydraulic head in Case 4 is higher than that in Case 2. Appropriate subsurface water usage makes water resources sustainable. Moreover, subsurface water overdraft causes land subsidence problems in many places; therefore preventing the consequences of aquifer exploitation is essential $[40,41]$. Results show that the minimum hydraulic head in Case 2 is around $73 \mathrm{~m}$ and that in Case 4 is around $81 \mathrm{~m}$ (Figure 9), representing that hydraulic head is under control using the FIS. As a result, fuzzy rules consider hydraulic head constraints implicitly for environmental conservation. Accordingly, the FIS is the intelligent control model based on the fuzzy rule and controls humanistic systems in water resources management. In the FIS approach, the rules with the expert experience can satisfy demand and environmental conservation adaptively. The FIS offers the ability for the adaptive management so that the system follows the fuzzy 
rule and adapts the supply water based on the states of the system. Thus, the managers can adjust the fuzzy operation strategy to satisfy the water demand and environmental conservation.

\section{Conclusions}

This study applied a fuzzy inference system (FIS) for the conjunctive use of surface and subsurface water. The FIS determines operating policies between surface and subsurface water based on the current states. The approach with the expert knowledge could obtain efficient and near-optimal solutions when compared to the simulation-optimization approach. After assigning the demand of surface and subsurface water, the ANN and LP simulate the surface and subsurface water states.

Results show that the FIS enhances reliability of water supply and provides a decision for utilizing two water sources. To minimize the impacts of consequential shortages, the FIS follows the operation rules in which abundant water system supplies more, but scarce water system supplies less. The FIS improves shortage performance because the FIS supplies subsurface water early and retains surface water during dry season. The FIS controls the supply between surface and subsurface water and reduces the impact of overpumping of subsurface water. Therefore, the FIS is best utilized in controlling humanistic systems whose behavior is influenced by expert knowledge for water resources management. Direction for future studies could consider an autotuning technology and a neural learning technology or parameter optimization approaches further acquiring the rule from expert knowledge [42].

\section{Acknowledgments}

The authors would like to thank C.W. Huang, P. C. Chen, editors, reviewers, and the anonymous helpers for their contributions to this study. In addition, the authors also thank Water Resources Agency, Department of Land Administration, Ministry of Interior and National Science Council, Taiwan, for financially supporting this research.

\section{References}

[1] L. A. Zadeh, "Fuzzy sets," Information and Control, vol. 8, no. 3, pp. 338-353, 1965.

[2] M. Negnevitsky, Artificial Intelligence: A Guide to Intelligent Systems, Addison Wesley, Reading, Mass, USA, 2002.

[3] B. P. Shrestha, L. Duckstein, and E. Z. Stakhiv, "Fuzzy rule-based modeling of reservoir operation," Journal of Water Resources Planning and Management, vol. 122, no. 4, pp. 262-268, 1996.

[4] S. O. Russell and P. F. Campbell, "Reservoir operating rules with fuzzy programming," Journal of Water Resources Planning and Management, vol. 122, no. 3, pp. 165-170, 1996.

[5] D. P. Panigrahi and P. P. Mujumdar, "Reservoir operation modelling with fuzzy logic," Water Resources Management, vol. 14, no. 2, pp. 89-109, 2000.
[6] M. Özger, "Comparison of fuzzy inference systems for streamflow prediction," Hydrological Sciences Journal, vol. 54, no. 2, pp. 261-273, 2009.

[7] H. Chu and L. Chang, "Application of optimal control and fuzzy theory for dynamic groundwater remediation design," Water Resources Management, vol. 23, no. 4, pp. 647-660, 2009.

[8] C. Mahabir, F. E. Hicks, and A. R. Fayek, "Application of fuzzy logic to forecast seasonal runoff," Hydrological Processes, vol. 17, no. 18, pp. 3749-3762, 2003.

[9] O. Kisi, M. E. Karahan, and Z. Şen, "River suspended sediment modelling using a fuzzy logic approach," Hydrological Processes, vol. 20, no. 20, pp. 4351-4362, 2006.

[10] H. Başağaoğlu and M. A. Mariño, "Joint management of surface and ground water supplies," Ground Water, vol. 37, no. 2, pp. 214-222, 1999.

[11] R. C. Peralta, R. R. A. Cantiller, and J. E. Terry, "Optimal large-scale conjuctive water-use planning: case study," Journal of Water Resources Planning \& Management, vol. 121, no. 6, pp. 471-478, 1995.

[12] C. R. Philbrick and P. K. Kitanidis, "Optimal conjunctive-use operations and plans," Water Resources Research, vol. 34, no. 5, pp. 1307-1316, 1998.

[13] S. V. N. Rao, S. M. Bhallamudi, B. S. Thandaveswara, and G. C. Mishra, "Conjunctive use of surface and groundwater for coastal and deltaic systems," Journal of Water Resources Planning and Management, vol. 130, no. 3, pp. 255-267, 2004.

[14] D. W. Watkins and D. C. McKinney, "Decomposition methods for water resources optimization models with fixed costs," Advances in Water Resources, vol. 21, no. 4, pp. 283-295, 1998.

[15] T. Nishikawa, "Water-resources optimization model for Santa Barbara, California," Journal of Water Resources Planning and Management, vol. 124, no. 5, pp. 252-263, 1998.

[16] M. N. Azaiez, "A model for conjunctive use of ground and surface water with opportunity costs," European Journal of Operational Research, vol. 143, no. 3, pp. 611-624, 2002.

[17] E. Coppola, Optimal pumping policy for a public supply wellfield using computational neural network with decision-making methodology [Ph.D. thesis], University of Arizona at Tucson, Tucson, Ariz, USA, 2000.

[18] E. Coppola, M. Poulton, E. Charles, J. Dustman, and F. Szidarovszky, "Application of artificial neural networks to complex groundwater management problems," Natural Resources Research, vol. 12, no. 4, pp. 303-320, 2003.

[19] E. Coppola, F. Szidarovszky, M. Poulton, and E. Charles, "Artificial neural network approach for predicting transient water levels in a multilayered groundwater system under variable state, pumping, and climate conditions," Journal of Hydrologic Engineering, vol. 8, no. 6, pp. 348-359, 2003.

[20] S. Feng, S. Kang, Z. Huo, S. Chen, and X. Mao, "Neural networks to simulate regional ground water levels affected by human activities,” Ground Water, vol. 46, no. 1, pp. 80-90, 2008.

[21] H. Chu and L. Chang, "Optimal control algorithm and neural network for dynamic groundwater management," Hydrological Processes, vol. 23, no. 19, pp. 2765-2773, 2009.

[22] A. W. Minns and M. J. Hall, "Artificial neural networks as rainfall-runoff models," Hydrological Sciences Journal, vol. 41, no. 3, pp. 399-417, 1996.

[23] H. R. Maier and G. C. Dandy, "Neural networks for the prediction and forecasting of water resources variables: a review of modelling issues and applications," Environmental Modelling and Software, vol. 15, no. 1, pp. 101-124, 2000. 
[24] J. Morshed and J. J. Kaluarachchi, "Application of artificial neural network and genetic algorithm in flow and transport simulations," Advances in Water Resources, vol. 22, no. 2, pp. 145-158, 1998.

[25] M. Tu, N. Hsu, and W. W.-G. Yeh, "Optimization of reservoir management and operation with hedging rules," Journal of Water Resources Planning and Management, vol. 129, no. 2, pp. 86-97, 2003.

[26] J. W. Labadie, "Optimal operation of multireservoir systems: state-of-the-art review," Journal of Water Resources Planning and Management, vol. 130, no. 2, pp. 93-111, 2004.

[27] Hydrologic Engineering Center (HEC), HEC-5 Simulation of Flood Control and Conservation Systems: User's Manual. Version 8, US Army Corps of Engineers, Davis, Calif, USA, 1998.

[28] C. Wei and N. Hsu, "Multireservoir real-time operations for flood control using balanced water level index method," Journal of Environmental Management, vol. 88, no. 4, pp. 1624-1639, 2008.

[29] MATLAB, Neural Network Tool Box for Use with Matlab: User Guide. Version 4, vol. 3, The Mathwork, Apple Hill Drive, Mass, USA, 2000.

[30] L. W. Mays and Y. K. Tung, Hydrosystems Engineering and Management, McGraw-Hill, New York, NY, USA, 1992.

[31] Hydrologic Engineering Center (HEC), Reservoir Yield, Generalized Computer Program 23-J2-L245, US Army Corps of Engineers, Davis, Calif, USA, 1966.

[32] Hydrologic Engineering Center (HEC), Hydrologic Engineering Methods for Water Resources Development, Volume 8, Reservoir Yield, US Army Corps of Engineers, Davis, Calif, USA, 1975.

[33] K. Ponnambalam, F. Karray, and S. J. Mousavi, "Minimizing variance of reservoir systems operations benefits using soft computing tools," Fuzzy Sets and Systems, vol. 139, no. 2, pp. 451461, 2003.

[34] J. W. Labadie, "Optimal operation of multireservoir systems: state-of-the-art review," Journal of Water Resources Planning and Management, vol. 130, no. 2, pp. 93-111, 2004.

[35] D. C. McKinney and M. -D. Lin, "Genetic algorithm solution of groundwater management models," Water Resources Research, vol. 30, no. 6, pp. 1897-1906, 1994.

[36] L. Chen, J. McPhee, and W. W.-G. Yeh, "A diversified multiobjective GA for optimizing reservoir rule curves," Advances in Water Resources, vol. 30, no. 5, pp. 1082-1093, 2007.

[37] L. Chang, H. Chu, and C. Hsiao, "Optimal planning of a dynamic pump-treat-inject groundwater remediation system," Journal of Hydrology, vol. 342, no. 3-4, pp. 295-304, 2007.

[38] A. W. Harbaugh and M. G. McDonald, "Programmer's documentation for MODFLOW-96, an update to the U.S. Geological Survey modular finite-difference ground-water flow model," Open-File Report 96-486, U.S. Geological Survey, Reston, Va, USA, pp. 220, 1996.

[39] P. E. Gill, W. Murray, and M. H. Wright, Practical Optimization, Academic Press, New York, NY, USA, 1981.

[40] O. G. Adrian, D. L. Roudolph, and J. A. Cherry, "Analysis of long-term land subsidence near Mexico City: field investigations and predictive modeling," Water Resources Research, vol. 35, no. 11, pp. 3327-3341, 1999.

[41] N. C. Don, N. T. M. Hang, H. Araki, H. Yamanishi, and K. Koga, "Groundwater resources and management for paddy field irrigation and associated environmental problems in an alluvial coastal lowland plain," Agricultural Water Management, vol. 84, no. 3, pp. 295-304, 2006.
[42] K. Ying, S. Lin, Z. Lee, and I.-L. Lee, "A novel function approximation based on robust fuzzy regression algorithm model and particle swarm optimization," Applied Soft Computing Journal, vol. 11, no. 2, pp. 1820-1826, 2011. 

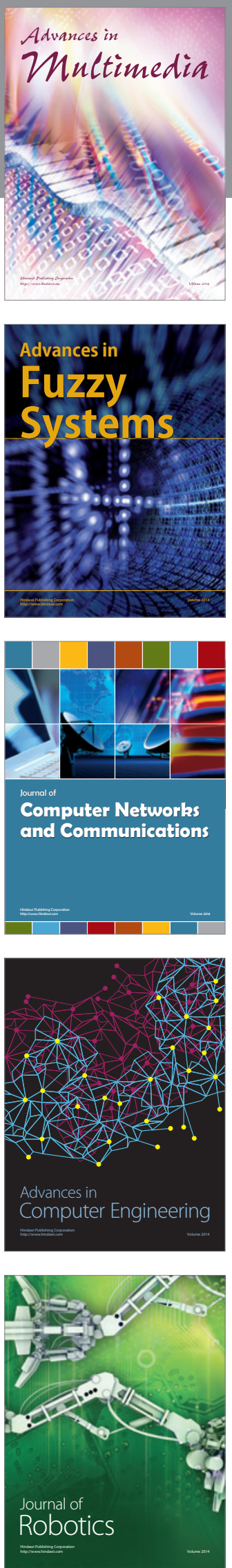

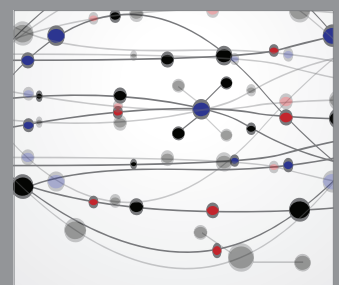

The Scientific World Journal
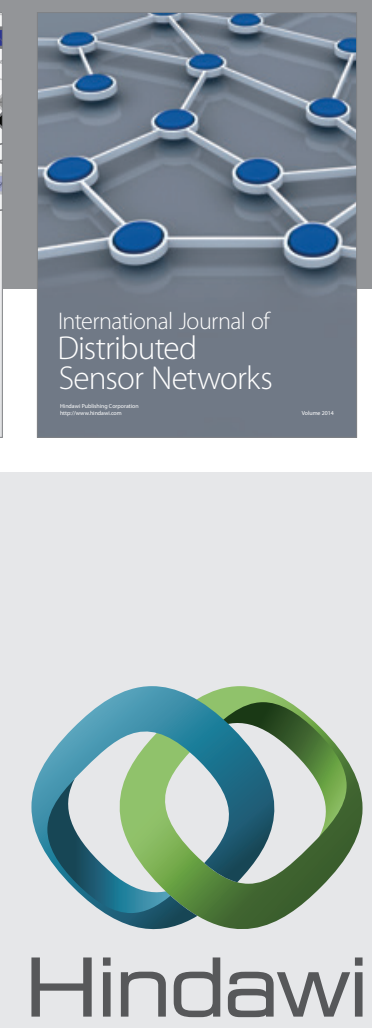

Submit your manuscripts at

http://www.hindawi.com
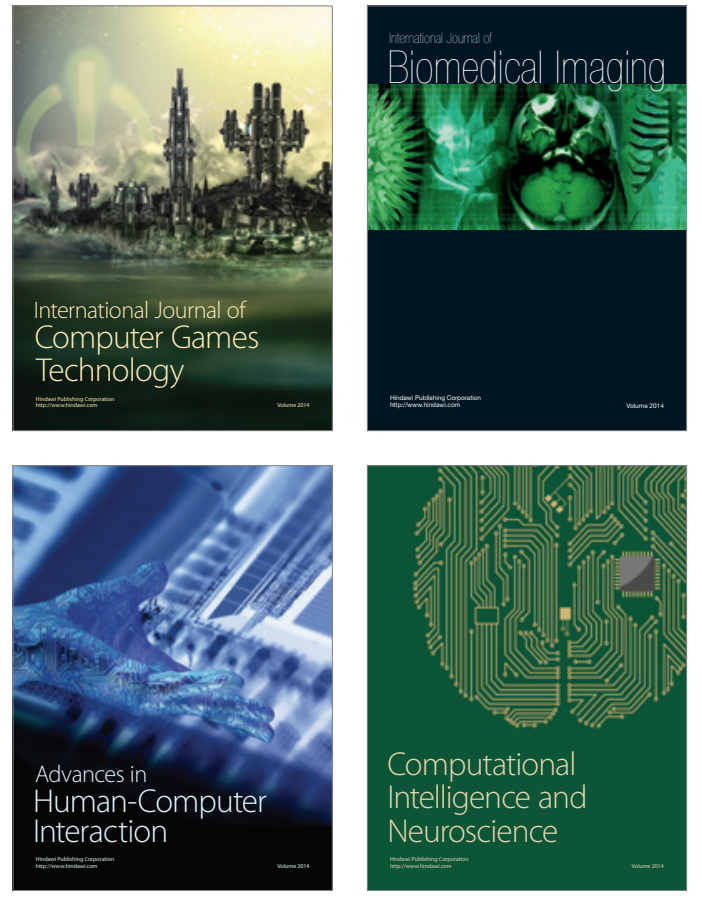
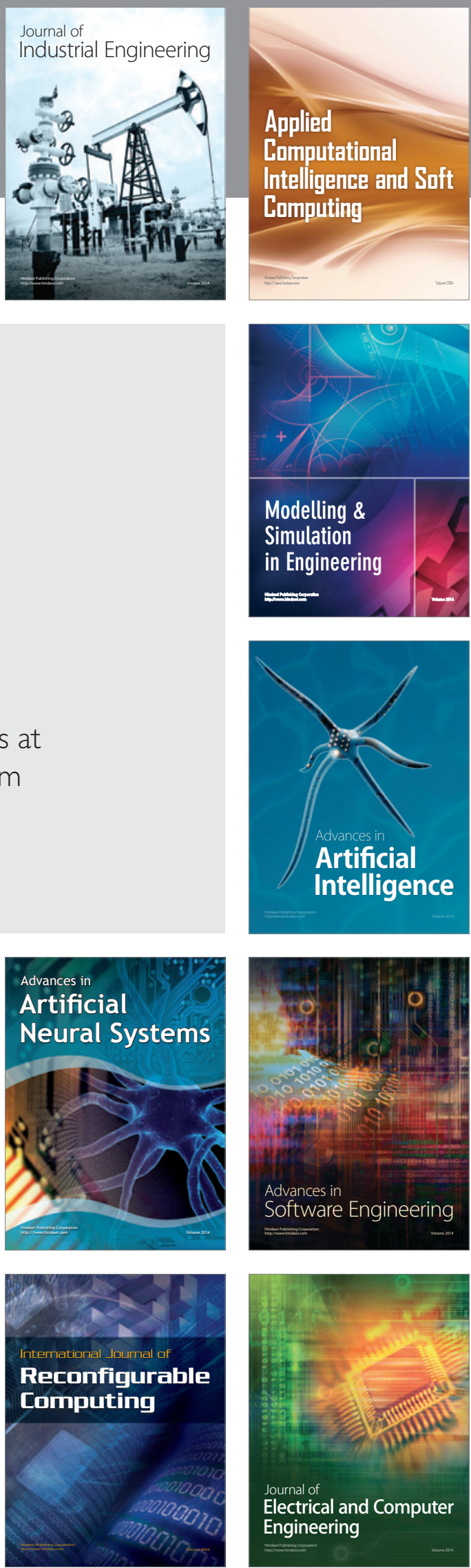